\title{
Força muscular e qualidade de vida em idosas
}

\author{
Muscular strength and quality of life in elderly women
}

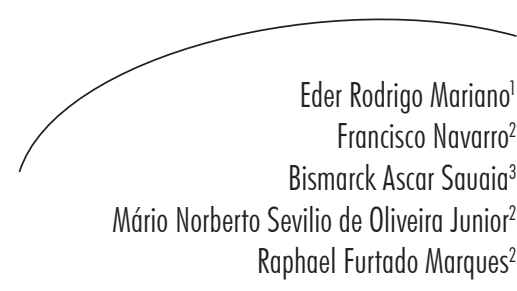

Resumo

Objetivo: Verificar o efeito do treinamento físico sobre os níveis de força e a qualidade de vida, considerando capacidade funcional, limitações físicas, dor, estado geral de saúde, vitalidade e variáveis de relação social em idosas institucionalizadas. Métodos: Participaram do estudo 36 idosas com 60 ou mais anos de idade, recrutadas no Serviço Social do Comércio (SESC), na cidade de São Luís-MA, compondo dois grupos: sedentário $(n=16)$ e treinamento $(n=20)$. Determinou-se a força muscular isométrica máxima dos extensores da coluna lombar e joelho, flexores de cotovelo e abdutores dos ombros, com o uso do dinamômetro NPRO2000. Aplicou-se questionário SF-36 para avaliação da qualidade de vida. O treinamento ocorreu em sessões, duas vezes por semana, de 60 minutos, por 12 semanas. A intensidade foi estabelecida pela zona de repetições máximas (três a quatro séries; 8 a 12 repetições) e a ordem dos exercícios foi modificada a cada quatro semanas. Estatisticamente, foi utilizado o teste $t$ Student do programa SPSS 10.0. Resultados: O grupo sedentário não alcançou valor significativo nas variáveis estudadas; o grupo treinamento atingiu escores significativos de ganho de força, nos extensores do joelho ( $\mathrm{p}=0,0032 ; 30,23 \%$ ) e extensores da coluna lombar $(\mathrm{p}=0,0207 ; 12,33 \%)$. A avaliação da qualidade de vida apresentou-se significativa, com aumento percentual nos domínios da capacidade funcional ( $\mathrm{p}=0,0092 ; 11,05 \%$ ), estado geral de saúde $(\mathrm{p}=0,0075 ; 14,17 \%)$, vitalidade $(\mathrm{p}=0,0015 ; 15,38 \%)$ e saúde mental $(\mathrm{p}=0,0154 ; 9,64 \%)$. Conclusão: $\mathrm{O}$ treinamento de força proposto promoveu aumento significativo na força muscular, repercutindo na melhoria da qualidade de vida nos domínios capacidade funcional, estado geral de saúde, vitalidade e saúde mental.

\footnotetext{
Departamento de Educação Física. Universidade CEUMA. São Luís, MA, Brasil.

2 Departamento de Educação Física, Centro de Ciências da Saúde. Universidade Federal do Maranhão. São Luís, MA, Brasil.

3 Laboratório Morfofuncional, Faculdade de Medicina. Universidade CEUMA. São Luís, MA, Brasil.
}

Correspondência / Correspondence

Eder Rodrigo Mariano

E-mail: mariano_eder@hotmail.com

Palavras-chave: Idoso. Feminino. Envelhecimento. Força Muscular. Qualidade de vida. 


\section{Abstract}

Objectives: To investigate the effect of exercise training on the levels of strength and quality of life in elderly women, considering the functional capacity, physical limitations, pain, general health, vitality and social relationship variables in institutionalized elderly. Methods: The study participants were 36 elderly women over 60 years old, recruited in the Social Service of Commerce (SESC), city of São Luís, state of Maranhão, Brazil, divided in tw groups: the sedentary group $(n=16)$ and training group $(n=20)$. All were 60 years or more, had not performed strength training for six months and had no restrictions on this practice. Before and after training, it was determined maximal isometric muscle strength of the extensors of the lumbar spine and knee, the elbow flexors and abductors of the shoulder, using the dynamometer NPRO2000. We used the SF-36 questionnaire to assess te quality of life. Training was conducted for 12 weeks with twice-weekly sessions of 60 minutes. The intensity was set by the zone of maximum repetitions (3-4 sets, 8-12 reps) and the order of exercises changed every four weeks. Statistical analysis was performed using the Student $t$ test program SPSS 10.0. Results: The sedentary group did not achieve any statistically significant value in any variable studied; the training group achieved statistically significant scores and percentage values of strength gain, respectively, in knee extensors $(\mathrm{p}=0.0032$, $30.23 \%$ ) and extensors of the lumbar spine ( $\mathrm{p}=0.0207 ; 12.33 \%)$. The results obtained in evaluating the quality of life showed statistically significant percentage increase in the functional capacity $(\mathrm{p}=0.0092 ; 11.05 \%)$, general health $(\mathrm{p}=0.0075 ; 14,17 \%)$, vitality $(\mathrm{p}=0.0015,15.38 \%)$ and mental health $(\mathrm{p}=0.0154 ; 9.64 \%)$ of elderly submitted to strength training. Conclusion: The strength training proposed in this study caused a significant increase in muscle strength that reflected in the improvement of quality of life in the domains of physical functioning, general health, vitality and mental health of elderly women submitted to strength training.
Key words: Elderly. Female. Aging. Muscle Strength. Quality of Life.

\section{INTRODUÇÃOO}

O processo de envelhecimento fisiológico promove uma diminuição das reservas funcionais do indivíduo, ${ }^{1}$ acarretando mudanças morfofisiológicas, funcionais e bioquímicas, tornando-o mais suscetível a agressões intrínsecas e extrínsecas. ${ }^{2}$ Dentre as principais alterações observadas, está o decréscimo da função muscular, que afeta diretamente a capacidade de realizar tarefas do dia a dia, diminuindo a independência funcional $\mathrm{e}$ refletindo negativamente na qualidade de vida do idoso. ${ }^{3}$

A sarcopenia ou síndrome da fragilidade caracteriza-se pela redução da massa muscular esquelética. ${ }^{4,5}$ Para Doherty, ${ }^{6}$ esse processo pode ser resultado da diminuição do número e/ou quantidade de fibras musculares dos tipos I e II, porém as fibras do tipo II, que possuem maior capacidade de gerar tensão e velocidade de encurtamento, são as mais atingidas pela sarcopenia.

Para Larsson, Grimby \& Karlsson, ${ }^{8}$ o maior índice de força muscular é alcançado por volta dos 30 anos, o qual se mantém estável até a quinta década, havendo redução na força muscular em torno de $15 \%$ entre a quinta e a sétima década, com acentuação ainda maior (30\%) após os 80 anos de idade.

A redução de massa muscular (sarcopenia) pode gerar limitações funcionais (dinapenia) que acarretam perda da independência, quedas e fraturas. ${ }^{910}$ As consequências negativas da 
sarcopenia e dinapenia na população idosa se expressam nos elevados índices de morbimortalidade, ${ }^{11,12}$ no aumento do número de assistências sociais e sanitárias ${ }^{13}$ e nos registros de incapacidade física de idosos. ${ }^{4,5,10}$

Os exercícios de força são indispensáveis em qualquer programa de condicionamento físico. ${ }^{14}$ Por meio do treinamento de força, Singh ${ }^{15}$ constatou que indivíduos com idade acima de 90 anos obtiveram ganhos de força e melhorias tanto na capacidade funcional quanto no aumento da mobilidade geral. Idosos submetidos ao treinamento de força apresentam aumento significativo na ativação, potência e aumento da massa e força muscular. ${ }^{7,16-21}$ Hernandes \& Barros $^{22}$ relataram ganho na capacidade funcional de idosos após a participação no programa de treinamento de força. Aveiro et.al. ${ }^{23}$ constatou que os exercícios físicos que visam o aumento da força muscular exercem efeitos benéficos sobre a qualidade de vida.

O aumento na expectativa de vida e a vulnerabilidade a doenças crônico-degenerativas e comorbidades remetem à busca de intervenções que minimizem os efeitos deletérios do envelhecimento, com consequente melhoria da qualidade de vida. ${ }^{24-28}$ Assim, a presente pesquisa objetivou verificar o impacto do treinamento de força, sua relação com a qualidade de vida, considerando a capacidade funcional, limitações físicas, dor, estado geral de saúde, vitalidade e variáveis de relação social em idosas institucionalizadas.

\section{METODOLOGIA}

\section{Sujeitos}

O estudo incluiu 36 mulheres idosas, sendo 20 do grupo treinamento e 16 do grupo sedentário, recrutadas aleatoriamente entre janeiro e junho de 2011, na Unidade Deodoro do Serviço Social do Comércio (SESC) em São Luís-MA, e convidadas a participar da pesquisa.
Os critérios de inclusão foram não restrição médica para a prática de exercícios de força e não ter participado de nenhum tipo de treinamento com pesos há, no mínimo, seis meses. Foram excluídas aquelas com restrição médica para a prática de exercícios de força.

\section{Força muscular}

$\mathrm{Na}$ determinação da força muscular isométrica máxima, utilizou-se o dinamômetro N2000PRO, da Biotecnologia Esportiva (CEFISE), assim como na demonstração dos resultados de força de pico, força média e máxima em (Kg/f) e Newton (N), em tempo real. Detectou-se a força de extensores do joelho, extensores da coluna lombar, abdutores do ombro e flexores de cotovelo, antes e após o protocolo de treinamento.

Qualidade de vida

$\mathrm{Na}$ avaliação da qualidade de vida, foi aplicado o questionário The Medical Outcomes Study 36 - Item Short - Form Health Survey (SF-36), ferramenta fidedigna para avaliação da capacidade funcional nas atividades diárias. ${ }^{29} \mathrm{O}$ SF-36 contempla oito domínios, sendo quatro relacionados ao componente físico: capacidade funcional, aspectos físicos, dor, estado geral de saúde; e quatro domínios relacionados ao componente mental: vitalidade, aspectos sociais, aspectos emocionais e saúde mental.

Tratamento estatístico

No tratamento estatístico, foi utilizado o teste t Student do software SPSS 10.0 para comparar os valores referentes aos índices de força muscular e de qualidade de vida obtidos antes e depois do treinamento, considerando o nível de confiança de $95 \%$. 
Princípios éticos

A pesquisa foi aprovada pelo Comitê de Ética do Hospital Universitário do Maranhão (CEP/ HU/UFMA) com o parecer consubstanciado $n^{\circ} 276 / 2011$ e executada em cumprimento aos princípios éticos da declaração de Helsinki, Resolução no 196/96 do Conselho Nacional de Saúde. Todos os participantes assinaram previamente o Termo de Consentimento Livre e Esclarecido (TCLE).

\section{RESULTADOS}

Os resultados obtidos na tabela 1 - grupo treinamento apresentaram valores estatísticos significativos, quando comparados os dois momentos da pesquisa (antes e depois do treinamento de força); portanto, o exercício aplicado melhorou significativamente a extensão do joelho e da coluna lombar.

Tabela 1 - Avaliação da força muscular de idosas assistidas em uma unidade do SESC, submetidas a exercícios de resistência de força. São Luís, MA, 2011.

\begin{tabular}{|c|c|c|c|c|c|c|c|c|}
\hline \multirow{3}{*}{$\begin{array}{c}\text { Variáveis } \\
\text { de } \\
\text { Avaliação } \\
\text { da Força }\end{array}$} & \multicolumn{8}{|c|}{ Exercícios de Resistência de Força } \\
\hline & \multicolumn{4}{|c|}{ Grupo Sedentário - n(20) } & \multicolumn{4}{|c|}{ Grupo Treinamento - n(16) } \\
\hline & $\begin{array}{c}\text { AT } \\
\text { Média } \pm \text { DP }\end{array}$ & $\begin{array}{c}\text { DT } \\
\text { Média } \pm \text { DP }\end{array}$ & (t) & (p) & $\begin{array}{c}\text { AT } \\
\text { Média } \pm \text { DP }\end{array}$ & $\begin{array}{c}\text { DT } \\
\text { Média } \pm \text { DP }\end{array}$ & $(\mathrm{t})$ & (p) \\
\hline $\begin{array}{l}\text { Extensão } \\
\text { do joelho }\end{array}$ & $28,05 \pm 6,87$ & $26,89 \pm 7,06$ & $+2,63$ & 0,0188 & $31,99 \pm 7,37$ & $41,66 \pm 10,62$ & $-3,37$ & 0,0032 \\
\hline $\begin{array}{l}\text { Extensão } \\
\text { lombar }\end{array}$ & $38,87 \pm 5,16$ & $3,68 \pm 4,17$ & $+2,40$ & 0,0299 & $41,85 \pm 10,31$ & $47,01 \pm 12,08$ & $-2,52$ & 0,0207 \\
\hline
\end{tabular}

$\mathrm{AT}=$ antes do treinamento; $\mathrm{DT}=$ depois do treinamento; $(\mathrm{t})=$ valor do teste.

Os resultados demonstrados na tabela 2-grupo treinamento, sobre a avaliação da qualidade de vida, revelaram valores estatísticos significativos após o treinamento, no que tange a capacidade funcional, estado geral de saúde, vitalidade, limitação de aspectos sociais e saúde mental. 
Tabela 2 - Avaliação da qualidade de vida de idosas assistidas em uma unidade do SESC, submetidas a exercícios de resistência de força. São Luís, MA, 2011.

\begin{tabular}{|c|c|c|c|c|c|c|c|c|}
\hline \multirow{4}{*}{$\begin{array}{l}\text { Variáveis de } \\
\text { Avaliação da } \\
\text { Qualidade } \\
\text { de Vida }\end{array}$} & \multicolumn{8}{|c|}{ Exercícios de Resistência de Força } \\
\hline & \multicolumn{4}{|c|}{ Grupo Sedentário - n(20) } & \multicolumn{4}{|c|}{ Grupo Treinamento - $\mathrm{n}(16)$} \\
\hline & AT & DT & & & AT & DT & & \\
\hline & Média \pm DP & Média \pm DP & (L) & (P) & Média \pm DP & Média \pm DP & (L) & (P) \\
\hline $\begin{array}{l}\text { Capacidade } \\
\text { funcional }\end{array}$ & $66,37 \pm 25,36$ & $61,44 \pm 27,42$ & $+3,64$ & 0,0024 & $72,35 \pm 20,07$ & $80,35 \pm 19,76$ & $-2,90$ & 0,0092 \\
\hline $\begin{array}{l}\text { Estado geral } \\
\text { de saúde }\end{array}$ & $71,62 \pm 21,46$ & $68,94 \pm 20,50$ & $+1,26$ & 0,2255 & $73,40 \pm 16,30$ & $83,80 \pm 11,51$ & $-2,99$ & 0,0075 \\
\hline Vitalidade & $\begin{array}{l}69,37 \pm \\
22,281\end{array}$ & $67,81 \pm 22,61$ & $+0,84$ & 0,4156 & $69,55 \pm 10,13$ & $80,25 \pm 10,94$ & $-3,70$ & 0,0015 \\
\hline $\begin{array}{l}\text { Saúde } \\
\text { mental }\end{array}$ & $71,56 \pm 20,94$ & $71,44 \pm 20,81$ & $+0,10$ & 0,9200 & $81,95 \pm 14,85$ & $89,85 \pm 11,09$ & $-2,66$ & 0,154 \\
\hline
\end{tabular}

$\mathrm{AT}=$ antes do treinamento; $\mathrm{DT}=$ depois do treinamento; $(\mathrm{t})=$ valor do teste.

\section{DISCUSSÃO}

O treinamento de força em idosos é um tipo de exercício que vem sendo apontado como eficaz no retardamento do declínio de força e massa muscular, comum ao processo de envelhecimento. ${ }^{14}$ Valores similares aos encontrados neste estudo foram detectados na investigação de Lima et. al., ${ }^{30}$ onde se observou aumento de $16,7 \%$ na avaliação isocinética e $54,7 \%$ na avaliação 1RM na força de quadríceps, em idosas submetidas a seis meses de treinamento com carga progressiva de $60 \%$ a $80 \%$ de $1 \mathrm{RM}$, com três séries de oito a 12 repetições. $\mathrm{O}$ programa de fortalecimento proposto por Rossi et al. ${ }^{31}$ expressou $\mathrm{p}=0,02$, referente ao ganho de força nos quadríceps de idosas, corroborando os achados no presente estudo.

A melhoria na qualidade de vida, considerando a capacidade funcional de idosas registrada por Martins, ${ }^{32}$ é semelhante a obtida na presente pesquisa. Engberg et al., ${ }^{33}$ em pesquisa com idosas dinamarquesas, documentaram os benefícios na dor, vitalidade e saúde emocional, com significância estatística somente na função física. Neste caso, no que tange à vitalidade, encontraram-se valores estatísticos significativos após o treinamento de força. No estudo de Vreede et al., ${ }^{34}$ os autores verificaram o efeito de tarefas funcionais e do exercício de resistênca sobre a qualidade de vida relacionada à saúde. A maioria das idosas apresentou melhorias no aspecto físico somente para o grupo de exercícios de resistência, ratificando a eficácia do treinamento de força sobre a capacidade funcional.

A respeito dos efeitos do treinamento de força sobre os aspectos mentais na qualidade de vida e concomitantemente aos resultados da pesquisa sobre a variável "saúde mental", destaca-se o estudo de Busse et al., ${ }^{35}$ que detectou resultados positivos e estatisticamente significativos no comportamento da memória de idosos sedentários que apresentavam déficit cognitivo, após participarem de um programa de força. Igualmente, Shay \& Roth ${ }^{36}$ evidenciaram a influência que a atividade física exerce sobre o estado geral de saúde, ratificando as afirmativas do presente estudo. 
A perda de segmento por evasão ou desistência, assim como a dificuldade em recrutar idosas para treinamento físico, provocou limitações no transcorrer dos trabalhos, superadas pelo acompanhamento amostral e melhoria da qualidade de vida das idosas submetidas ao exercício de força.

\section{CONCLUSÃO}

Sobre os resultados obtidos na pesquisa, considerando o treinamento de força, foi

\section{REFERÊNCIAS}

1. Cristopoliski F, Barela JA, Leite N, Fowler NE, Rodacki AL. Stretching exercise program improves gait in the elderly. Gerontology 2009;55(6):614-20

2. Barbosa SM, Arakaki J, Silva MF. Estudo do equilíbrio em idosos através de fotogrametria. Fisioter Bras 2001;2(3):189-96

3. Lacourt MX, Marini LL. Decréscimo da função muscular decorrente do envelhecimento e a influência na qualidade de vida do idoso: uma revisão de literatura. RBCEH - Rev Bras Ci Env Hum 2006;3(1):114-21

4. Goodpaster BH, Park SW, Harris TB, Kritchevsky SB, Nevitt M, Schwartz AV, et al. The loss of skeletal muscle strength, mass, and quality in older adults: The health, aging and body composition study. J Gerontol A Biol Sci Med Sci 2006;61(10):1059-64.

5. Delmonico MJ, Harris TB, Lee JS, Visser M, Nevitt M, Kritchevsky SB, et al. Alternative definitions of sarcopenia, lower extremity performance, and functional impairment with aging in older men and women. J Am Geriatr Soc 2007;55(5):769-74.

6. Doherty TJ. Invited review: aging and sarcopenia. J Appl Physiol (1985) 2003;95(4):1717-27.

7. Izquierdo M, Aguado X, Gonzalez R, López JL, Häkkinen K. Maximal and explosive force production capacity balance performance in men of different ages. Eur J Appl Physiol Occup Physiol 1999;79(3):260-7.

8. Larsson L, Grimby G, Karlsson J. Muscle strength and speed of movement in relation to age and muscle morphology. J Appl Physiol Respir Environ Exerc Physiol 1979;46(3):451-6. possível concluir que: (a) as alterações inerentes ao processo de envelhecimento, como a sarcopenia e a dinapenia, são minimizadas por meio da prática de exercícios físicos; (b) os exercícios de força são fundamentais na prevenção e no combate à evolução de doenças crônico-degenerativas não transmissíveis que comprometem a capacidade funcional e a autonomia do idoso; e (c) o treinamento de força proposto na pesquisa melhorou a força muscular assim como a qualidade de vida das idosas, nos domínios capacidade funcional, estado geral de saúde, vitalidade e saúde mental.

9. Rosenberg IH. Sarcopenia: origins and clinical relevance. J Nutr 1997;127(5):990S-1S.

10. Janssen I, Heymsfield SB, Ross R. Low relative skeletal muscle mass (sarcopenia) in older persons is associated with functional impairment and physical disability. J Am Geriatr Soc 2002;50(5):889-96.

11. Sayer AA, Dennison EM, Syddall HE, Gilbody HJ, Phillips DI, Cooper C. Type 2 diabetes, muscle strength, and impaired physical function: the tip of the iceberg? Diabetes Care 2005;28(10):2541-2.

12. Gale CR, Martyn CN, Cooper C, Sayer AA. Grip strength, body composition, and mortality. Int J Epidemiol 2007;36(1):228-35.

13. Janssen I, Shepard DS, Katzmarzyk PT, Roubenoff $\mathrm{R}$. The healthcare costs of sarcopenia in the United States. J Am Geriatr Soc 2004;52(1):80-5.

14. American College of Sports Medicine. American College of Sports Medicine position stand. Progression models in resistance training for healthy adults. Med Sci Sports Exerc 2009;41(3):687-708.

15. Singh MA. Exercise comes of age: rationale and recommendations for a geriatric exercise prescription. J Gerontol A Biol Sci Med Sci 2002;57(5):M262-82.

16. Häkkinen K, Häkkinen A. Neuromuscular adaptations during intensive strength training in middle-aged and elderly males and females. Electromyogr Clin Neurophysiol 1995;35(3):137-47.

17. Häkkinen K, Alen M, Kallinen M, Izquierdo M, Jokelainen K, Lassila $\mathrm{H}$, et al. Muscle CSA, force production, and activation of leg extensors during isometric and dinamic actions in middle-aged and elderly men and woman. J Aging Phys Act 1998;6:232-47. 
18. Häkkinen K, Alen M, Kallinen M, Newton RU, Kraemer WJ. Neuromuscular adaptation during prolonged strength training, detraining and restrength-training in middle-aged and elderly people. Eur J Appl Physiol 2000;83(1):51-62.

19. Häkkinen K, Pakarinen A, Kraemer WJ, Häkkinen A, Valkeinen H, Alen M. Selective muscle hypertrophy, changes in EMG and force, and serum hormones during strength training in older women. J Appl Physiol (1985) 2001;91(2):569-80.

20. Häkkinen K, Kraemer WJ, Pakarinen A, TriplettMcBride T, McBride JM, Häkkinen A, et al. Effect of heavy resistance/power training on maximal strength, muscle morphology, and hormonal response patterns in 60-75 year-old men and women. Can J Appl Physiol 2002;27(3):213-31.

21. Nogueira W, Gentil P, Mello SN, Oliveira RJ, Bezerra AJ, Bottaro M. Effects of power training on muscle thickness of older men. Int J Sports Med 2009;30(3):200-4.

22. Hernandes ESC, Barros JF. Efeitos de um programa de atividades físicas e educacionais para idosos sobre o desempenho em testes de atividades da vida diária. R Bras Ci e Mov 2004 jun;12(2):43-50.

23. Aveiro MC, Navega MT, Granito RN, Rennó ACM, Oishi J. Efeitos de um programa de atividade física no equilíbrio e na força muscular do quadríceps em mulheres osteoporóticas visando uma melhoria na qualidade de vida. R Bras Ci e Mov 2004;12(3):33-8.

24. Fleck MPA, Leal OF, Louzada S, Xavier M, Chanchamovich E, Vieira G, et al. Desenvolvimento da versão em português do instrumento de avaliação de qualidade de vida da OMS (WHOQOL-100). Rev Bras Psiquiatr 1999;21(1):19-28.

25. Neri AL, Yassuda MS, organizadoras. Velhice bemsucedida: aspectos afetivos e cognitivos. Campinas: Papirus; 2004. 224 p.

26. Carvalho JAM, Garcia RA. O envelhecimento da população brasileira: um enfoque demográfico. Cad Saúde Pública 2003;19(3):725-33.

27. Wong LLR, Carvalho JA. O rápido processo de envelhecimento populacional do Brasil: sérios desafios para as políticas públicas. Rev bras Est Pop 2006 jun;23(1):5-26.
28. Christensen K, McGue M, Petersen I, Jeune B, Vaupel JW.. Exceptional longevity does not result in excessive levels of disability. Proc Natl Acad Sci USA 2008;105(36):13274-19.

29. Costa D, Palma A. O efeito do treinamento contra resistência na síndrome da dor lombar. Rev Port Cien Desp 2005;5(2):224-34.

30. Lima RM, Bottaro M, Carregaro R, Oliveira JF, Bezerra LMA, Oliveira RJ. Efeitos do treinamento resistido sobre a força muscular de idosas: uma comparação entre métodos. Rev Bras Cineantropom Desempenho Hum 2012;14(4):409-18.

31. Rossi LP, Brandalize M, Gomes ARS. Efeito agudo da técnica de reeducação postural global na postura de mulheres com encurtamento da cadeia muscular anterior. Fisioter Mov 2001;24(2):255-63.

32. Martins RA, Fernandes FLF, Osório RAL, Ribeiro W. Relação da qualidade de vida associado ao treinamento resistido medida por meio do SF36. EFDeportes.com Rev Digital Buenos Aires [periódico na Internet]. 2012 [acesso em 28 maio 2011];15(166):[aproximadamente 3 p.]. Disponível em: http://www.efdeportes.com/efd166/treinamentoresistido-por-meio-do-sp-36.htm.

33. Engberg H, Oksuzyan A, Jeune B, Vaupel JW, Christensen K. Centenarians--a useful model for healthy aging? A 29-year follow-up of hospitalizations among 40,000 Danes born in 1905. Aging Cell 2009;8(3):270-6.

34. de Vreede PL, van Meeteren NL, Samson MM, Wittink HM, Duursma SA, Verhaar HJ. The effect of functional tasks exercise and resistance exercise on health-related quality of life and physical activity. A randomised controlled trial. Gerontology 2007;53(1):12-20.

35. Busse AL, Jacob Filho W, Magaldi RM, Coelho VA, Melo AC, Betoni RA, et al. Efeitos dos exercícios resistidos no desempenho cognitivo de idosos com comprometimento da memória: resultados de um estudo controlado. Einstein 2008;6(4):402-7.

36. Shay KA, Roth DL. Association between aerobic fitness and visuospatial performance in healthy older adults. Psychol Aging 1992;7(1):15-24. 